\title{
Growth temperature dependence of VLS-grown ultra-long ZnS nanowires prepared by CVD method
}

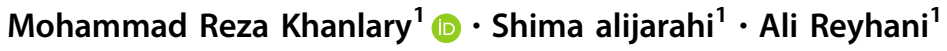

Received: 8 March 2018 / Accepted: 2 July 2018/Published online: 12 July 2018

(C) The Author(s) 2018

\begin{abstract}
The synthesis of ultra-long high-quality zinc sulfide $(\mathrm{ZnS})$ nanowires of uniform size on heterogeneous substrates is highly desirable for investigating the fundamental properties of $\mathrm{ZnS}$ nanowires and for fabricating integrated functional nanodevices. The present study developed a novel technique for growing ultra-long $\mathrm{ZnS}$ nanowires on thin-catalyst-coated substrates. $\mathrm{ZnS}$ nanowires were synthesized by chemical vapor deposition on a silicon substrate deposited by gold ( $\sim 5 \mathrm{~nm}$ in thickness) as the catalyst at $550,600,700,750$ and $800{ }^{\circ} \mathrm{C}$. The structural properties of the samples were investigated by X-ray diffraction, and the results showed that the fabricated nanowires have both wurtzite and zinc blend structures. The morphological properties of the nanowires were determined by scanning electron microscopy, and the results show that the substrate is thoroughly coated with $10 \mu \mathrm{m}$ of zinc sulfide nanowires. Increasing the substrate temperature from 600 to $800{ }^{\circ} \mathrm{C}$ increased the diameter of the nanowires and decreased the length. The growth mechanism of the nanowires was vapor-liquid-solid. The EDX spectra of this sample showed an absence of contamination, confirming the high purity of the $\mathrm{ZnS}$ nanowires.
\end{abstract}

Keywords Zinc sulfide $(\mathrm{ZnS}) \cdot$ Nanowire $\cdot$ Chemical vapor deposition · Morphology

\section{Introduction}

Semiconductor nanostructures have attracted great interest for studding the quantum confinement effect and thermodynamic structures of these materials [1-3]. Varieties of physical properties such as mechanical strength, plasticity [4], melting [5, 6],sintering and alloying ability [7], diffusivity [8], chemical reactivity [9], as well as the mode of crystal growth (self-assembly) [10], have been found dependent upon particle size. Zinc sulfide $(\mathrm{ZnS})$ with a wide direct optical band gap $\left(E_{\mathrm{g}} \sim 3.72 \mathrm{eV}\right)$ for cubic zinc blend (ZB) and $E_{\mathrm{g}} \sim 3.77 \mathrm{eV}$ for hexagonal wurtzite (WS) phase is an important II-VI semiconductor with a number of interesting properties. Nanostructured zinc sulfide materials have been intensively studied due to their wide potential applications in high-performance devices, such as optoelectronics, sensors, transducers and

Mohammad Reza Khanlary

khanlary@yahoo.com

1 Department of Physics, Imam Khomeini International University, Qazvin 34149-16818, Iran biomedical sciences [11]. As a one-dimensional nanostructure, $\mathrm{ZnS}$ has been synthesized as nanowires, nanobelts and nanocombs [12]. One of the most attracting features of nanowires (NWs) is that lattice mismatch or strain in NWs can be significantly relaxed due to their high surface/volume ratio and small lateral size. Different synthetic approaches have been used for the synthesis of $\mathrm{ZnS}$ nanomaterial, especially nanowires and nanorods; these methods include laser ablation [13], vapor transport in the presence [14] or in the absence of [15] catalyst, chemical vapor deposition (CVD) [16], electrochemical deposition [17] and solvothermal method [18]. Among them, CVD has got a number of advantages such as simplicity and the ability to use a wide variety of precursors such as liquid precursors, gaseous precursors, solid precursors which include halides, hybrids, metal-organic and organic. It also enables the usage of a variety of substrates and allows materials growth in a variety of forms, such as powder, thin or thick films aligned or entangled straight or coiled nanotubes. It also offers better control on the growth process of materials [19]. CVD allows proper control on the deposition rate and pressure temperature so as to prepare and 
maintain the desired structure, composition and size of the materials. Growth of nanowires via the vapor-liquid-solid (VLS) mechanism is an effective method, in which the diameter and length of nanowires are also affected by other growth conditions such as the growth ratio and temperature. [20]. In $\mathrm{ZnS}$ nanowire preparation by VLS, a thin layer of $\mathrm{Au}$ is used as the catalyst by thermal evaporation of $\mathrm{ZnS}$ powder [21]. However, separate evaporation of sulfur and zinc powder for $\mathrm{ZnS}$ nanowire preparation, which is more controllable by sulfur activity, is rarely reported. In the present work, we have preferred this route of synthesis and successfully prepared very long $\mathrm{ZnS}$ nanowires.

\section{Experimental}

The $\mathrm{ZnS}$ nanowires were synthesized by vapor-liquidsolid (VLS) technique. The conventional VLS mechanism is based on evaporation of the source material and transfer of the vapor to lower-temperature regions using an inert gas as a carrier. The experimental setup (CVD system in Fig. 1) consisted of a horizontal tube furnace $(6 \mathrm{~cm}$ in diameter; $50 \mathrm{~cm}$ in length) with a temperature control unit, a horizontal quartz tube $(4.5 \mathrm{~cm}$ in diameter; $102 \mathrm{~cm}$ in length 102), two alumina boats, a rotary vacuum pump, 5-nm-thick Au-coated n-type Si (with a preferential plane of 400) substrates and Ar gas as the carrier gas with a gas flow control system and a barometer. The alumina boat containing sulfur powder ( $1 \mathrm{~g}, 99 \%$ purity; Merck) and the boat containing granular zinc $(0.8 \mathrm{~g} ; 99.9 \%$ purity; Merck) were kept at 140 and $950{ }^{\circ} \mathrm{C}$, respectively. The Au-coated Si substrates were kept at 550, 600, 700, 750 and $800{ }^{\circ} \mathrm{C}$ in the center of the furnace. One terminal of the quartz tube was connected to a vacuum pump and gas flow system. The rotary vacuum pump evacuated the tube to a base pressure of $5 \times 10^{-2}$ mbar.

After closing the rotary pump, the tube was filled with high-purity Ar gas to eliminate any $\mathrm{O} 2$ in the tube, and the furnace was turned on to heat up. When the furnace reached the process temperature $\left(950^{\circ} \mathrm{C}\right)$, the terminal opposite to the gas flow system opened and was kept open during the process to allow the gas to flow out at a rate of 50 SCCM (sample A) and 80 SCCM (sample B). The pressure inside the tube was increased to $\sim 2 \times 10^{-1} \mathrm{mb}$, and the Ar carrier gas was fed into it. The values for flow rate and dynamic vacuum were kept constant until the end of the experiment. The surface morphology of the nanowires was investigated by means of SEM. To investigate the chemical composition of the $\mathrm{ZnS}$ nanowires, elemental analysis of the samples was performed using energy-dispersive X-ray (EDX) spectroscopy.

\section{Results and discussion}

Three distinct stages existed in the catalyst-assisted growth of $\mathrm{ZnS}$ nanowires. In the first stage, the Au film-coated Si substrate is annealed at $\sim 700{ }^{\circ} \mathrm{C}$. The Au film aggregated to form small islands because of the liquid-like mobility of the $\mathrm{Au}$ atoms at high temperature. In the second stage, called alloying and nucleation, the islands were exposed to $\mathrm{Zn}$ vapor carried in $\mathrm{Ar}$ gas and reacted with the vapor to form an $\mathrm{Au}-\mathrm{Zn}$ alloy of increased volume. At $950{ }^{\circ} \mathrm{C}$, the vapor pressure of zinc was $>10^{3}$ mbar, which was much greater than the sulfur vapor pressure at $140{ }^{\circ} \mathrm{C}$ of $\sim 10^{-1}$ mbar and system pressure of $2 \times 10^{-1} \mathrm{mbar}$.

Sulfur vapor and $\mathrm{Zn}$ vapor were continuously absorbed by alloy droplets and reacted with each other inside the droplet until zinc sulfide reached supersaturation. At this stage, the $\mathrm{ZnS}$ precipitated in the interface with the substrate and alloy droplets to form zinc sulfide nanocrystals. Next, $\mathrm{ZnS}$ nanowires were grown with Au nanoparticles in top of them. It can be said that the $\mathrm{ZnS}$ nanowire growth mechanism was VLS. It can be seen that the size and position of the catalyst relate to the diameter and position of the nanowires, as the liquid phase was confined to the area of the precipitated solid phase. Figure 2 shows SEM images of samples $\mathrm{A}$ and $\mathrm{B}$ at a substrate temperature of $550{ }^{\circ} \mathrm{C}$. A low Ar flow rate (50 SCCM) in Fig. 2a produced
Fig. 1 Schematic diagram of CVD unit

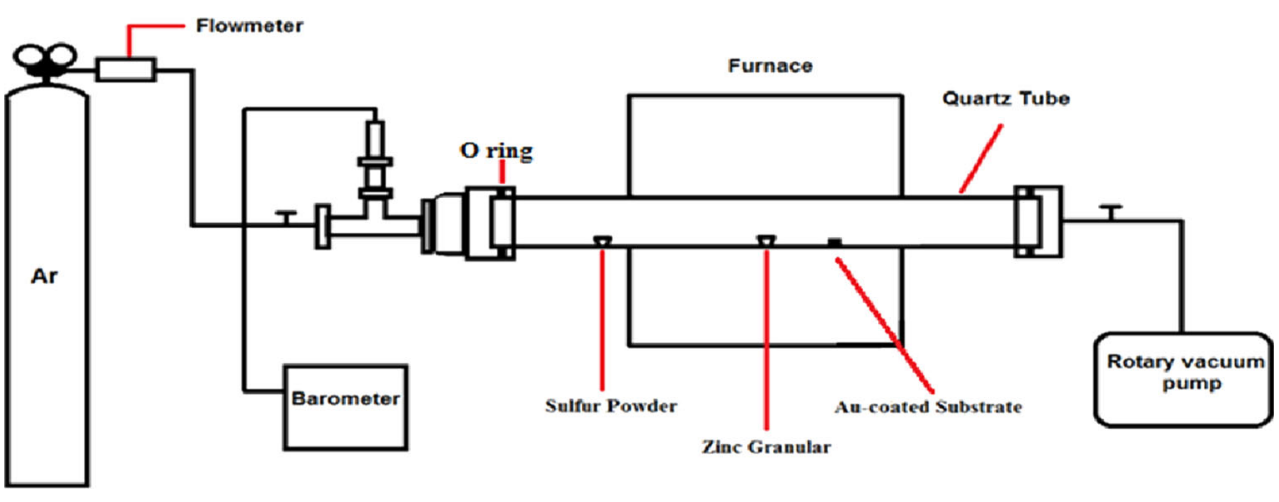


(a)

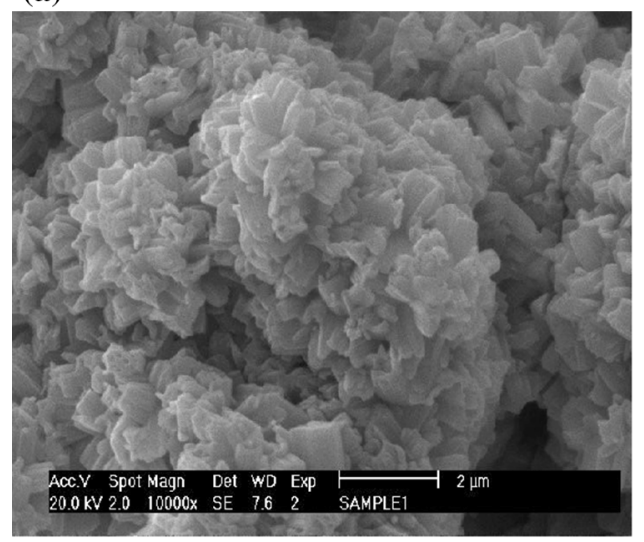

(b)

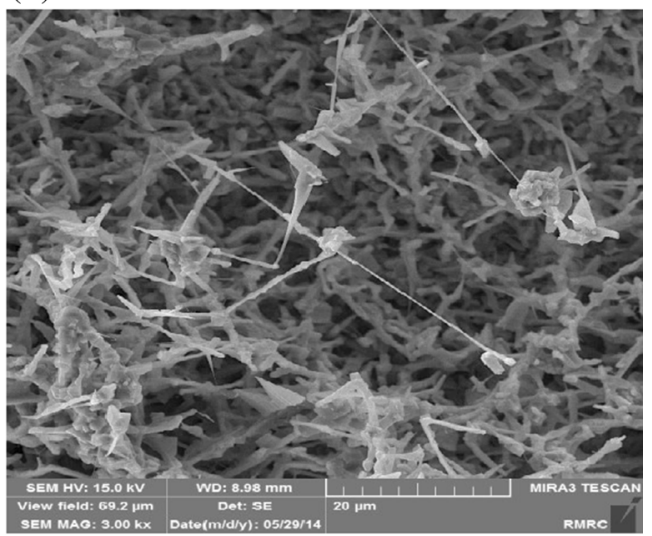

(c)

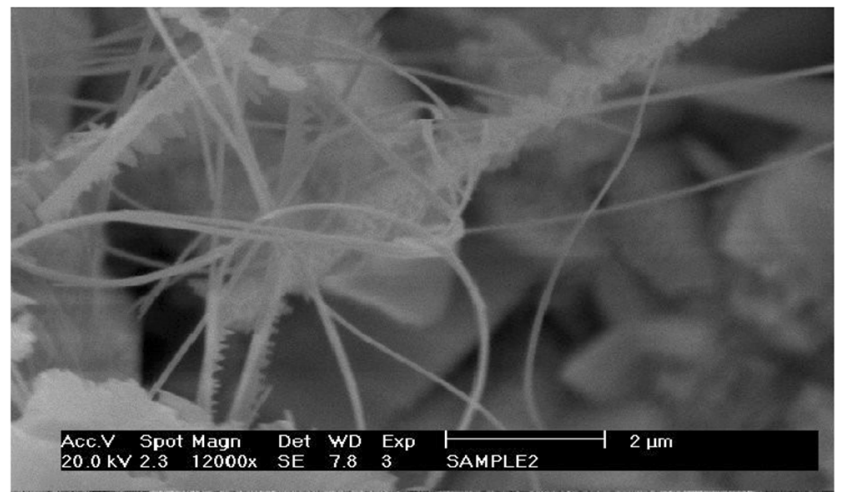

Fig. 2 SEM images of $\mathrm{ZnS}$ nanowires grown on Au-coated Si substrate at $550{ }^{\circ} \mathrm{C}$ : a low, $\mathbf{b}$ high Ar flow rate and $\mathbf{c}$ sample prepared with a low flow rate at substrate temperature of $600{ }^{\circ} \mathrm{C}$

no wire, but formed nanoflowers. At a higher gas flow rate in Fig. 2b, both long straight rods and shapeless structures formed.

Figure $2 \mathrm{c}$ is a sample prepared under the same conditions as in Fig. 2a with the addition of a substrate at $600{ }^{\circ} \mathrm{C}$. This shows that the synthesized products consisted of a small quantity of nanowires with diameters of about $60-100 \mathrm{~nm}$ and a length of several $\mu \mathrm{m}$ along with nanocombs and tetrapodal nanostructures.

The chemical potential of the component in the metal alloy catalyst increased as the size of the catalyst decreased because of the Gibbs-Thomson effect. Dissolving a vapor component into a liquid alloy became increasingly difficult as the size decreased, making it difficult to reach a sufficient supersaturation state to induce the growth of nanowires. It is known that the growth of nanowires with diameters of more than $>10 \mathrm{~nm}$ is feasible; however, growing those $<10 \mathrm{~nm}$ in size is difficult because of the thermodynamic limitation associated with the use of a catalyst.

Figure 3a, c, e shows that the substrate was densely covered with randomly oriented nanowires. The diameters of the nanowires grown at 700,750 and $800{ }^{\circ} \mathrm{C}$ were about 70-120, 80-160 and 100-190 nm, respectively. Under high magnification, the SEM images of these samples (Fig. 3b, $d, f)$ show Au nanoparticles on the tips of the nanowires in a VLS growth mechanism. An increase in growth temperature increased the average diameter of the $\mathrm{ZnS}$ nanowires grown on the $\mathrm{Si}$ substrates, and their average length decreased. Very long $\mathrm{ZnS}$ nanorods of $>10 \mu \mathrm{m}$ can be seen in all SEM images; however, straight rods were more abundant in the image in Fig. 3e than in 3a. As the substrate temperature increased to $800{ }^{\circ} \mathrm{C}$, a change from nanowires to nanorods occurred. In this mechanism, substrate temperature was the critical experimental parameter influencing the morphology and size of the nanostructures.

According to the VLS mechanism, the size of the Au nanoparticles plays a significant role in determining the length and diameter of the $\mathrm{ZnS}$ nanowires. The reports show that as the substrate temperature increases, the merging of Au nanoparticles increases [12]. It appears that the increase in average diameter and the decrease in average length relate to the increase in the diameter of $\mathrm{Au}$ nanoparticles.

The XRD patterns of nanowires grown on a Si substrate coated with Au film at 550, 600 and $700{ }^{\circ} \mathrm{C}$ demonstrate the temperature dependence of formation of $\mathrm{ZnS}$ nanowires (Fig. 4). The results indicate that an increase in growth 
(a)

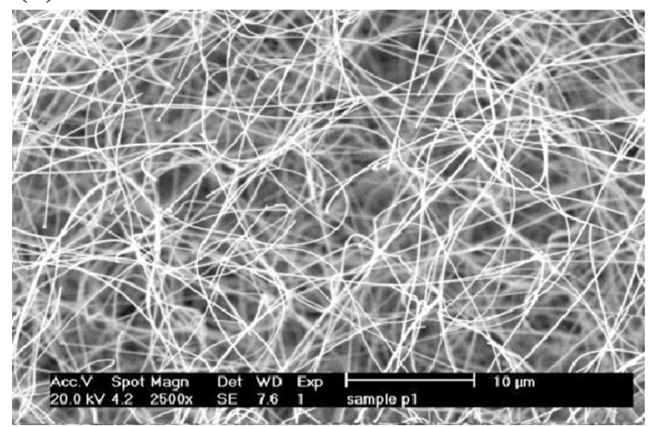

(c)

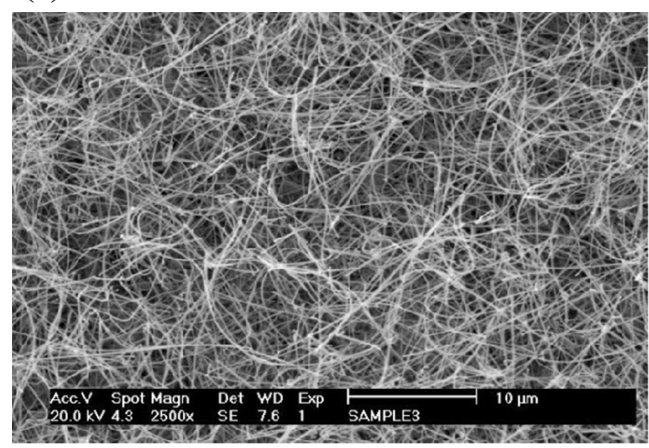

(e)

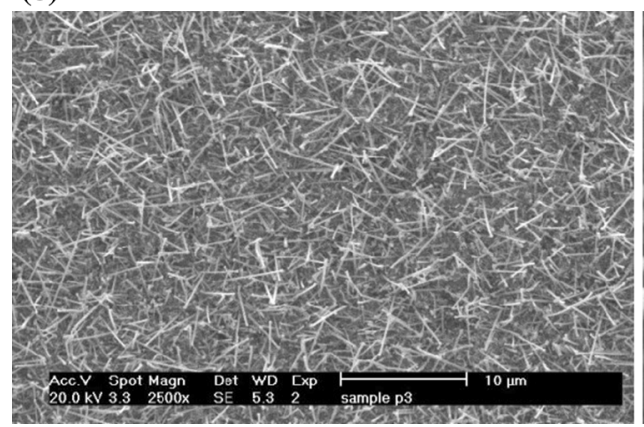

(b)

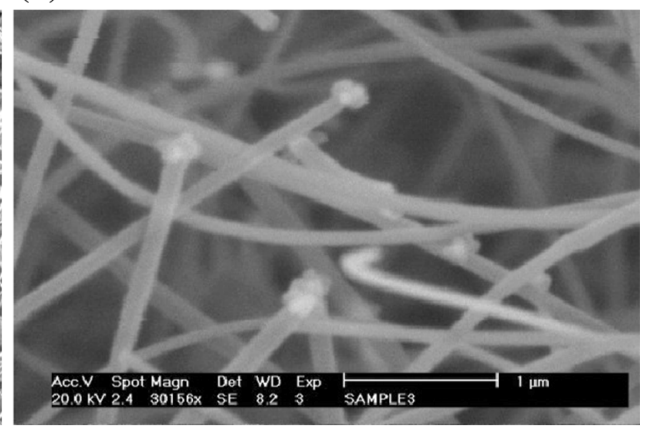

(d)

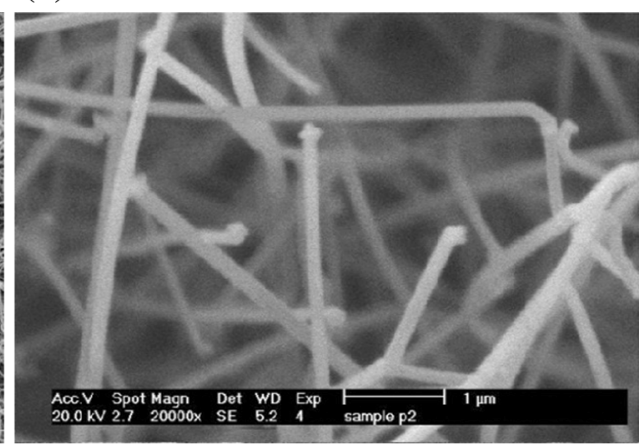

(f)

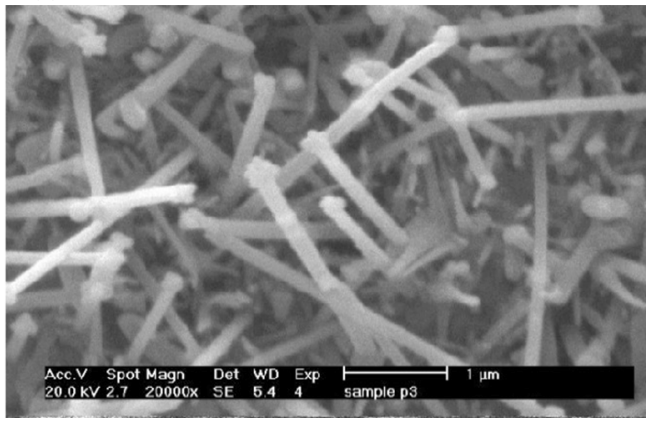

Fig. 3 SEM images at different magnifications of $\mathrm{ZnS}$ nanowires grown on Au-coated Si substrate at: $\mathbf{a}-\mathbf{b} 700{ }^{\circ} \mathrm{C} ; \mathbf{c}-\mathbf{d} 750{ }^{\circ} \mathrm{C}$ and $\mathbf{e}-\mathbf{f} 800{ }^{\circ} \mathrm{C}$

temperature increased the content of the wurtzite phase of nanowires grown on $\mathrm{Si}$ substrate. This is evident in the intensity of the (101) and (100) peaks of $\mathrm{ZnS}$ relative to the (200) peak of Si. The $\mathrm{ZnS}$ nanowires consisted of a matrix of hexagonal and cubic stacking of the $\mathrm{Zn}-\mathrm{S}$ layers according to standard cards (00-005-0492) and (00-0020565), respectively.

Diffraction peaks with $2 \theta$ values of $28.50,47.50$ and 56.30 related to the (111), (220) and (311) of the cubic phase of $\mathrm{ZnS}$ appeared. Moreover, XRD peaks at $2 \theta$ values of $26.90,30.50,39.60$ and 51.70 related to reflection from the (100), (101), (102) and (103) planes of wurtzite $\mathrm{ZnS}$ also appeared. The peak at $2 \theta$ of 32.90 relates to the Bragg reflection from the (200) plane of the silicon substrate. Two other features at $2 \theta$ values of 38.20 and 44.40 relate, respectively, to the (111) and (002) planes of the $\mathrm{Au}$ catalyst.

The small difference in Gibbs free energy [14] facilitates the phase transformation and is very sensitive to the reaction parameters. As the substrate temperature was quite high, both phases of the $\mathrm{ZnS}$ nanostructures were created in the mixture. The II-VI NWs entwined with the mixed ZB/ WS structures can be commonly observed along the (111) direction, such as $\mathrm{ZnS}$, [22] ZnSe [23] and ZnTe [24]. It is known that the cubic to hexagonal phase transformation takes place at a temperature of $<1020^{\circ} \mathrm{C}$, which is the cubic-hexagonal phase transition temperature of bulk $\mathrm{ZnS}$.

It is interesting to note that no $\mathrm{ZnO}$-related peaks were observed in the XRD patterns. A peak denoting $\mathrm{Au}_{2} \mathrm{~S}$ [depicted as (002) at a $2 \theta$ of 44.5] was observed. 


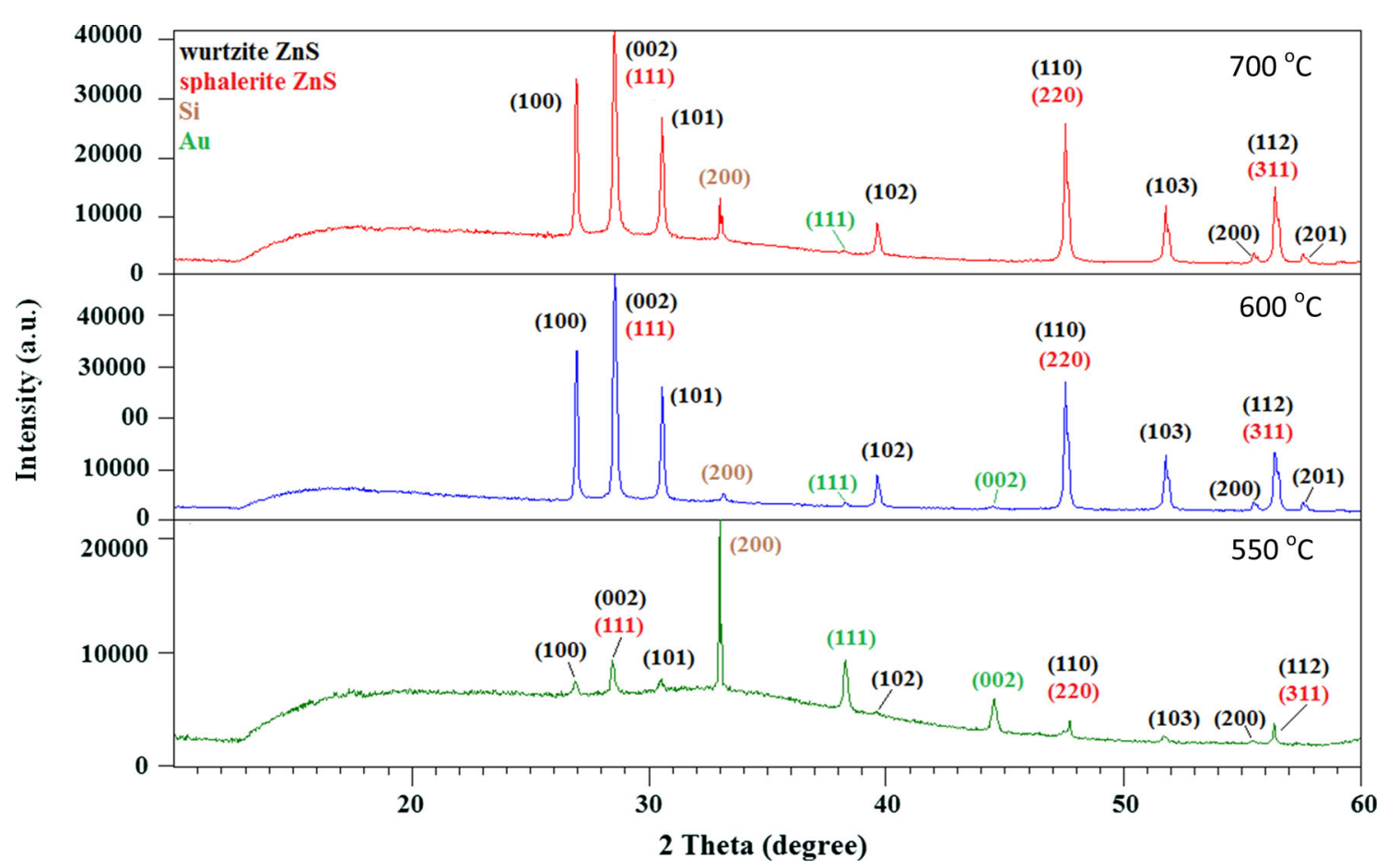

Fig. 4 XRD pattern of $\mathrm{ZnS}$ nanowires deposited on glass substrate at 550, 600 and $700{ }^{\circ} \mathrm{C}$

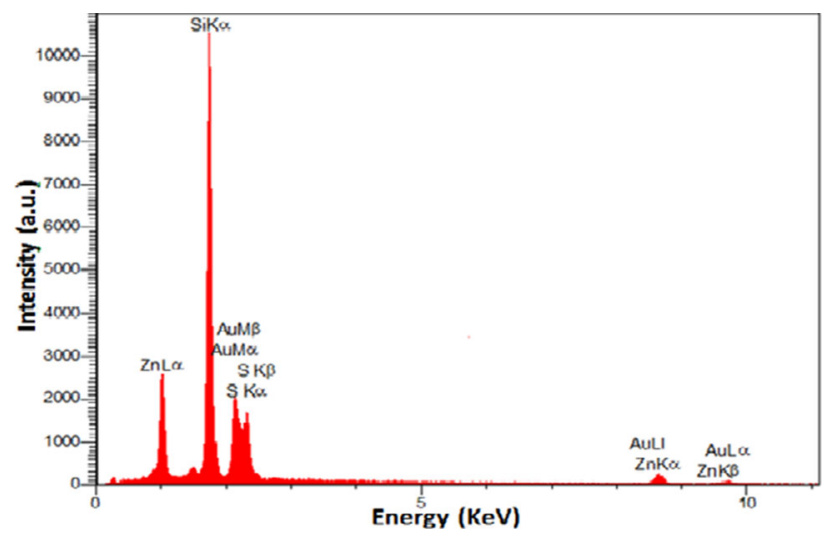

Fig. 5 Typical EDS spectra of $\mathrm{ZnS}$ nanowires grown on Au-coated Si substrate

Quantitative analysis shows that the quantity of this phase dropped significantly with an increase in annealing temperature of the Au catalyst from 550 to $700{ }^{\circ} \mathrm{C}$. It appears that the temperature dependence of energy of the Au catalyst and host caused a considerable drop in the concentration of the catalyst during the growth of the $\mathrm{ZnS}$ nanowires. This compound perhaps forms on the surface of the nanowires. The EDS spectra showed that $\mathrm{S}$ and $\mathrm{Zn}$ had atomic ratios of $42-58 \%$ (Fig. 5). Other peaks in the spectra relate to the Au-coated Si substrate.

\section{Conclusion}

A novel successive multistep growth method was developed to synthesize very long $\mathrm{ZnS}$ nanowires of several hundred $\mu \mathrm{m}$ in length. A systematic study was carried out to determine the optimal parameters affecting the dimensions of the nanowires synthesized on a large scale. Ultralong $\mathrm{ZnS}$ nanowires were grown successfully through thermal evaporation of zinc and sulfur powder at $600-800$ and $140{ }^{\circ} \mathrm{C}$, respectively. The $\mathrm{ZnS}$ nanowires were grown with a mixture of zinc blend and wurtzite structures. In VLS deposition, adjusting the synthesis conditions controls the formation of $\mathrm{ZnS}$ nanostructures as nanoflowers, nanowires and nanorods. Increasing the substrate temperature from 600 to $800{ }^{\circ} \mathrm{C}$ increases the nanowire diameter and decreases their length. The abundance of wurtzite structures in the mixture increased as the substrate temperature increased. XRD data analysis [as per the ratio of (100)-(002) peaks at different substrate temperatures] confirms that a cubic to hexagonal phase transformation has taken place at a lower temperature than that for bulk $\mathrm{ZnS}$.

Acknowledgements Authors would like to acknowledge from Imam Khomeini international university for their financial help.

Open Access This article is distributed under the terms of the Creative Commons Attribution 4.0 International License (http://creative 
commons.org/licenses/by/4.0/), which permits unrestricted use, distribution, and reproduction in any medium, provided you give appropriate credit to the original author(s) and the source, provide a link to the Creative Commons license, and indicate if changes were made.

\section{References}

1. Sheng, X., Gautam, U.K., Bando, Y., Dierre, B., Sekiguchi, T., Golberg, D.: Multiangular branched $\mathrm{ZnS}$ nanostructures with needle-shaped tips: potential luminescent and field-emitter nanomaterial. J. Phys. Chem. C 112(12), 4735-4742 (2008)

2. Ma, L.A., Wei, Z.H., Ye, X.Y., Lin, J.Y., Hu, L.Q., Guo, T.L.: Morphology-controlled synthesis and field-emission properties of patterned $\mathrm{SnO}_{2}$ nanostructures with different morphologies. Ceram. Int. 43(8), 6096-6104 (2017)

3. MaZ, L.A., Wei, H.: Field emission properties of crystalline$\mathrm{SnO}_{2}$ (core)/amorphous- $\mathrm{SnO}_{2}$ (shell) nanowire arrays on carbon paper. Mater. Lett. 213, 257-261 (2018)

4. Kudinova, N.R., Polyanskiy, V.A., Polyanskiy, A.M., Yakovlev, Y.A.: Modeling of the plasticity of microstructured and nanostructured materials. St. Petersb. Polytech. Univ. J. Phys. Math. 1(4), 405-409 (2015)

5. Nabeel Dhaidan, S.: Nanostructures assisted melting of phase change materials in various cavities. Appl. Therm. Eng. 111, 193-212 (2016)

6. Li, X.L., Wang, C.X., Yang, G.W.: Thermodynamic theory of growth of nanostructures. Prog. Mater Sci. 64, 121-199 (2014)

7. Mintova, S., Olson, N.H., Valtchev, V., Bein, T.: Mechanism of zeolite a nanocrystal growth from colloids at room temperature. Science 283, 958-960 (1999)

8. So Espen, S., Anders, D.B., Bo, B.B., Iversen, B.: Tailoring band gap and thermal diffusivity of nanostructured phase-pure $\mathrm{ZnAl}_{2} \mathrm{O}_{4}$ by direct spark plasma sintering synthesis. J. Solid State Chem. 256, 45-52 (2017)

9. Sayle, T.X.T., Sayle, D.C.: Visualizing the enhanced chemical reactivity of mesoporous Ceria; simulating templated crystallization in silica scaffolds at the atomic level. J. Am. Chem. Soc. 136(10), 4056-4065 (2014)

10. Berbezier, I., De Crescenzi, M.: Self-assembly of nanostructures and nanomaterials. Beilstein J. Nanotechnol. 6, 1397-1398 (2015)

11. Wu, P., He, Y., Wang, H.F., Yan, X.P.: Conjugation of glucose oxidase onto Mn-doped $\mathrm{ZnS}$ quantum dots for phosphorescent sensing of glucose in biological fluids. Anal. Chem. 82(4), 1427-1433 (2010)
12. Fang, X., Zhai, T., Gautam, U.K., Li, L.G., Wu, L., Bando, Y., Golberg, D.: ZnS nanostructures: from synthesis to applications. Prog. Mater Sci. 56, 175-287 (2011)

13. Jiang, Y., Meng, X.M., Liu, J., Hong, Z.R., Lee, C.S., Lee, S.T.: $\mathrm{ZnS}$ nanowires with wurtzite polytype modulated structure. Adv. Mater. 13, 1195-1198 (2003)

14. Meng, X.M., Liu, J., Jiang, Y., Chen, W.W., LeeI Bello, C.S., Lee, S.T.: Structure- and size-controlled ultrafine $\mathrm{ZnS}$ nanowires. Chem. Phys. Lett. 382, 434-438 (2003)

15. Yang, Y., Zhang, W.: Preparation and photoluminescence of zinc sulfide nanowires. Mater. Lett. 58, 3836-3838 (2004)

16. Zhang, H., Zhang, S., Zuo, M., Li, G., Hou, J.: Synthesis of ZnS nanowires and assemblies by carbothermal chemical vapor deposition and their photoluminescence. Eur. J. Inorg. Chem. 2005, 47-50 (2005)

17. Xu, X.J., Fei, G.T., Yu, W.H., Wang, X.W., Chen, L., Zhang, L.D.: Preparation and formation mechanism of $\mathrm{ZnS}$ semiconductor nanowires made by the electrochemical deposition method. Nanotechnology 17, 426-429 (2006)

18. Chai, L., Du, J., Xiong, S., Li, H., Zhu, Y., Qian, Y.J.: Synthesis of quartzite $\mathrm{ZnS}$ nanowire bundles using a solvothermal technique. Phys. Chem. C 111, 12658-12662 (2007)

19. Wang, C., He, Y., Peng, C., Wang, S., Liu, X.: Controllable synthesis of $\mathrm{Ni}$-catalyzed tetragonal tungsten nanowires via chemical vapor deposition. Prog. Nat. Sci. Mater. Int. 22(5), 514-519 (2012)

20. Zhu, G., Zhou, Y., Wang, S., Yang, R., Ding, Y., Wang, X., Bando, Y., Lin Wang, Z.: Synthesis of vertically aligned ultralong $\mathrm{ZnO}$ nanowires on heterogeneous substrates with catalyst at the root. Nanotechnology 23, 055604-055609 (2012)

21. Lin, M., Sudhiranjan, T., Boothroyd, C., Ping Loh, K.: Influence of Au catalyst on the growth of $\mathrm{ZnS}$ nanowires. Chem. Phys. Lett. 400, 175-178 (2004)

22. Hao, Y., Meng, G., Wang, Z., Ye, C., Zhang, L.: Periodically twinned nanowires and polytypicnanobelts of $\mathrm{ZnS}$ : the role of mass diffusion in vapor-liquid-solid growth. Nano Lett. 6, 1650 (2006)

23. Wang, Y., Phillpose, U., Xu, T., Ruda, H., Khavanagh, K.: Twinning modulation in ZnSe nanowires. Semicond. Sci. Technol. 22, 175 (2007)

24. Meng, Q., Jiang, C., Mao, S.: Temperature-dependent growth of zinc-blende-structured ZnTe nanostructures. J. Cryst. Growth 310, 4481 (2008)

Publisher's Note Springer Nature remains neutral with regard to jurisdictional claims in published maps and institutional affiliations. 